\title{
Is Swaziland census data suitable for fertility measurement?
}

\author{
Garikayi B. Chemhaka ${ }^{1,2^{*}}$, Clifford Odimegwu ${ }^{1 \dagger}$, Eugene N. Zwane ${ }^{2 \dagger}$ and Jeremy D. Gumbo ${ }^{1+}$
}

\author{
* Correspondence: bgchem@gmail. \\ com \\ ${ }^{\dagger}$ Equal contributors \\ ${ }^{1}$ Demography and Population \\ Studies Programme, Schools of \\ Social Sciences and Public Health, \\ University of the Witwatersrand, \\ Private Bag 3, Wits 2050 \\ Johannesburg, South Africa \\ ${ }^{2}$ Department of Statistics and \\ Demography, Faculty of Social \\ Sciences, University of Swaziland, \\ Private Bag 4, Kwaluseni M201, \\ Swaziland
}

\begin{abstract}
This study seeks to assess fertility data in Swaziland based on the 1976, 1986, 1997 and 2007 censuses. By utilising single-year age-sex published raw data, demographic evaluation tools - sex ratio, age ratio, Whipple's index, and modified Whipple's index-were used to assess age misreporting as several fertility measures rely on the quality of age data. In addition, using published descriptive census tables for women in the reproductive lifespan, 15-49, data on children ever born or parity $(P)$ derived were evaluated for incorrect reporting of parities using the el-Badry technique. Further, the relational Gompertz model was applied to adjust data on reported aggregated births in the last year or current fertility $(F)$ relying on its intrinsic $P / F$ ratios feature as an adjustment and diagnostic tool for consistency checks on fertility and parity distributions. The evidence of some age reporting distortions or age misreporting is not too severe, but moderate, and therefore fertility estimates in the four censuses of Swaziland cannot be said are invalidated. The data on parity and current fertility conforms to expected or typical patterns of fertility distributions of African populations. The study concludes that the census data were of reasonable quality for fertility estimation.

Keywords: Age-sex distribution, Census, Fertility, Data quality, Swaziland
\end{abstract}

\section{Introduction}

The efforts in evaluating and monitoring the past 2015 Millennium Development Goals (MDGs) has been so crucial for most African countries towards achieving set targets. The post-MDGs era now entails the realisation of the need of reliable data so crucial for developmental planning.

The article examines the importance of evaluating fertility data prior to estimation as utilising poor quality data leads to deriving implausible estimates of fertility. This in turn affects the decision-making process in development and policy planning which results in designing inappropriate programme interventions. The paper focuses on evaluating age and sex data and fertility data on children ever born (parity) and recent births reported for past years in censuses. Recent births reflect current fertility whilst parity data provide lifetime fertility measures.

Whilst the position on quality of demographic data in censuses also surveys has been contested and investigated using various demographic and statistical tools for some Southern African countries such as South Africa (Moultrie and Timæus 2002; Phillips 1999), Zimbabwe and Botswana (Blanc and Rutstein 1994; Thomas and Muvandi

(c) 2016 The Author(s). Open Access This article is distributed under the terms of the Creative Commons Attribution 4.0 International License (http://creativecommons.org/licenses/by/4.0/), which permits unrestricted use, distribution, and reproduction in any medium, provided you give appropriate credit to the original author(s) and the source, provide a link to the Creative Commons license, and indicate if changes were made. 
1994), such scientific enquiry is missing for Swaziland in spite of regular census enumerations done. Thus, fertility data remains under-examined for Swaziland. Further, the in-depth analysis of Swaziland census data has been limited due to paucity of data. Previous actual raw data sets such as for 1976 and 1986 are not in the reach or easily accessible for public use. The articles seek to examine the suitability of Swaziland 1976, 1986, 1997 and 2007 census data for deriving plausible fertility estimates by bringing to the fore data quality issues when deriving fertility estimates. Fertility was about 7 children per woman in the 1960s (Cohen 1993) and was 4 at the 2006/7 Demographic and Health Survey (DHS) and 2007 census.

As a preliminary step, census data are evaluated on accuracy and quality of age reporting errors using classic demographic tools such as age ratios, sex ratios and Myers', Whipple's and joint score indices on pinpointing irregularities on age-sex composition (Brass 1996; Shryock et al. 1976; Spoorenberg 2007). Also employed are the diagnostic tools of the $P / F$ ratios inherent in the relational Gompertz model and the el-Badry correction method to evaluate fertility data. The multiple census data sources allows for consistency checks on age-sex distributions and comparisons of fertility data (Joyner et al. 2012; Moultrie et al. 2013; United Nations 2004) amongst the four censuses of Swaziland. Therefore, the purpose is to demonstrate, but not necessarily quantify, the possible errors that may arise from estimating fertility using Swaziland census data. This study focuses on consistency checks using average parity, age and sex distributions and age pattern of fertility as basis for evaluating fertility data derived from the four censuses.

In contrary to less flawed demographic data for developed countries, for African countries in the past four decades, the data has been deemed as either defective or inadequate with respect to its poor quality (Cleland 1996; Cohen 1993, 1998; Brass 1996; Moultrie et al. 2013; United Nations 1983; Potter 1977; Hill 1990). Due to an incomplete vital registration system (VRS) in most developing countries, the census, therefore, remains as the reliable and main source of fertility as well as mortality measurement (United Nations 2004). Indeed a census is not an exceptional stand-alone or alternative data source to a VRS or survey, but it is indispensable and complementary for demographic measurement.

In deriving fertility estimates, the potential errors in census data are numerous or inescapable. The types, causes and effects of errors on estimates of fertility are highlighted in demographic literature (National Research Council 2004; Schoumaker 2014; Vergauwen et al. 2015; Potter 1977; Brass 1996; Joyner et al. 2012; Cleland 1996; Spoorenberg 2014; Phillips 1999; United Nations 1983, 2004; Hill 1990; Moultrie et al. 2013). This literature highlights common quality issues on age-sex and fertility data which relate to omission, duplication, misreporting, mistiming or misplacement of birth events or age, selection bias and non-responsiveness or response irregularities due to memory lapse or recall problems and/or negligence as well as other motives in reporting/recording. A distorted age-sex structure through bias and errors in age-sex data has implications on fertility estimates and their projections (Spoorenberg 2014). The United Nations (1983) notes that the effect of age misreporting on fertility is complex, but the overall effect tends to be small.

In overview, it is difficult to uncover, distinguish or correct all errors in data and hence the concern is on reducing and knowing their bias or overall effect (National 
Research Council 2004; Schoumaker 2014). Errors (of different types) which may have occurred on a simultaneous basis could lead to a similar effect on fertility (Schoumaker 2014) or an offset (National Research Council 2004; Shryock et al. 1976). Further, possible effects of errors or bias, in magnitude or direction, which Brass (1996) cautions their difficulty in proving, can be compensated by adjustments using appropriate methods of applications and assumptions in fertility estimation (Hill 1990; Moultrie et al. 2013). For example, information on parities $(P)$ and current fertility $(F)$ has led to utilisation of a resourceful demographic tool-lifetime/current fertility $(P / F)$ ratio. This ratio is vital to evaluation of fertility data and estimation of fertility using many indirect methods derived on this principle such as the preferable relational Gompertz model (Moultrie et al. 2013). Thus, the United Nations (2004) and Moultrie et al. (2013) posit that though data may portray errors on some aspects, it may be possible to come up with robust levels and trends of fertility.

\section{Data and methods}

The data for this study are based on published raw and aggregated census tabulations in the 1976, 1986, 1997 and 2007 censuses undertaken by the Central Statistics Office (CSO) of Swaziland. Published raw data on population distribution by age and sex were used. Tabulated aggregated demographic data collected from census reports were fertility variables on recent births and children ever born. The question on children ever born was asked from women over some minimum age, 12 or 15 years in the censuses.

Consistent with modern census questioning on fertility, the four censuses in Swaziland are similar in content. Although raw data for 1976 and 1986 censuses are not accessible, published aggregate data on age, sex and fertility are credible for appraisal of census data quality.

With the available raw data, in the 2007 census, $20 \%$ of the sample has missing "unknown" or "non-stated" parities needed for assessing and correcting parity distributions, whereas the full data set for 1997 census has adequate fertility variables. To maintain consistency on data use, single-year and aggregated data were derived from descriptive tables of published census reports for the total population. The methods used to evaluate age-sex and fertility data in the four censuses are explained in the next sections.

\section{Quality of age-sex data}

Classic demographic ratios and indices such as sex ratios, age ratios, Whipple's index and Myers' blended index are the several methods used for the appraisal of age-sex data for errors in age misreporting and digit preference or avoidance (Shryock et al. 1976; Moultrie et al. 2013; Spoorenberg 2007). The discussion on methods which follows draws extensively from these authors.

The sex ratio, defined as the number males for every 100 females, is one simple measure of evaluating age-sex data by merely observing its deviation from 100, a point defining equal size of males and females. The overall sex ratio largely depends on the population age distribution. An expected range of deviation from 100 should be accounted for by changes in the population such as migration and mortality. Consistency of sex composition can be verified when the sex ratio in one census is compared to that of the previous census. In most populations, the sex ratio at birth 
(SRB) is expected to be above 100 signifying excess male over female births. The SRB is generally estimated or assumed to be 105 and can be expected to be lower in African populations closer to 103 or 100 in some cases. The sex ratio (SR) for a given single age $x$ is expressed mathematically as:

$$
\mathrm{SR}=P_{x}^{m} / P_{x}^{f} \times 100
$$

where $P_{x}^{m}$ and $P_{x}^{f}$ refer to male and female population at age $x$, respectively. Moultrie et al. (2013) opined that in the age range 0 to about 45 years a typical sex ratio for developing countries declines gradually with increasing age with exception of excess net migration, especially amongst young adults. A steep decline occurs at older ages when male mortality is in excess of females.

The age ratio is obtained as a quotient of the population in a particular age group divided by the average population of the two adjacent age groups. The age ratio (AR) is expressed as follows:

$$
\mathrm{AR}={ }_{5} P_{x}^{5} /\left(0.5 \times\left({ }_{5} P_{x-5-5}{ }^{5}+{ }_{5} P_{x+5_{x+5}}{ }^{5}\right)\right) \times 100
$$

where ${ }_{5} P_{x}$ refers to the population at age $x$ to $x+5,{ }_{5} P_{x-5}$ and ${ }_{5} P_{x+5}$ represent the preceding and successive age groups of population, respectively. Like the sex ratio at each age group, the deviation from 100 implies net age misreporting. This can be expected as selective under-enumeration, over-enumeration or misclassification of age and a combination of one or more. A glitch in the age ratio often ignored is that the omission of the central age in the denominator leads to its upward bias.

Both internal consistency (meeting typical patterns on age and or sex distribution) and external consistency (comparison of data across censuses from the same country) are vital in data assessment (Moultrie et al. 2013). Therefore, for both age and sex ratios, an age range from birth to older ages was assessed with the expectation that an oldest 49-yearold woman in the 1976 census would be around 80 years in the 2007 census.

Whipple's index is utilised for single-year age data to show the extent of heaping on certain ages, usually 0 or 5 in the age range 23 to 62 (Shryock et al. 1976). Whipple's index $(W)$ is given as:

$$
W=100 \times 5 \sum_{x=25,5}^{60} P_{x} / \sum_{x=23}^{62} P_{x}
$$

where $\sum_{x=23}^{62} P_{x}$ refers to the summation of population in single-year completed ages $x$ from 23 to 62 and $\sum_{x=25,5}^{60} P_{x}$ stands for the summation of population completed singleyear ages $x$ in multiples of 5 from 25 to 60, that is, for ages with digits ending with 0 and 5. For its normal range of 100 to 500, a value of 100 indicates no heaping and 500 massive heaping. A value which is lower than 100 suggests heaping on certain ages other than 0 and 5 . Between the extreme values of 100 and 500, the quality of data is graded on a scale of accurate, approximate and rough representation.

The 23-62 age range for Whipple's index was altered to 23-52 to represent women in the reproductive lifespan for each census data under the same linearity assumptions. The digits 0 and 5 in the numerator are distributed evenly or linearly over 5 -year ages in the denominator, with the exception of ages lower than 23 and older ages greater than 62 (Shryock et al. 1976; Spoorenberg 2007) or 52 in the case of this study. A woman aged 49 probably would be able to respond to an age of 50 in a census due to a 0 digit preference. 
According to Spoorenberg (2007), Whipple's index is that the extent of digit preference is meant for terminal digits 0 and 5 . Age heaping at any age other than 0 or 5 may occur which can be detected using Myers' blended index. The index can be applied to an age range of 10-89 for all terminal digits, 0-9 (Shryock et al. 1976).

Since a shorter age range for women aged 15-49 is considered in this context, a Whipple-type version of Myers' index would be considered since it uses the same principles of Myers' index. Spoorenberg (2007), following the work proposed in 1992 by Noumbissi of modifying Whipple's index to detect age heaping at age 0 and 5 separately, extended the index to each terminal digit, $i$, for 0 to 9 and hence the term "digit-specific modified Whipple's index" $\left(W_{i}\right)$. Based on Spoorenberg (2007) algebraic expressions of $W_{i}$, an age range of 21-52 was considered representing women in the reproductive lifespan for each census.

The modification on Spoorenberg equations on $W_{i}$ for each terminal digit 0 to 9 limited to the age range $21-52$ is expressed as follows:

$$
\begin{aligned}
& W_{0}=5\left(P_{30}+P_{40}+P_{50}\right) /\left({ }_{5} P_{28}{ }^{5}+{ }_{5} P_{38}{ }^{5}+{ }_{5} P_{48}{ }^{5}\right) \\
& W_{1}=5\left(P_{31}+P_{41}\right) /\left({ }_{5} P_{29}{ }^{5}+{ }_{5} P_{39}{ }^{5}\right) \\
& W_{2}=5\left(P_{32}+P_{42}\right) /\left({ }_{5} P_{30}{ }^{5}+{ }_{5} P_{40}{ }^{5}\right) \\
& W_{3}=5\left(P_{23}+P_{33}+P_{43}\right) /\left({ }_{5} P_{21}{ }^{5}+{ }_{5} P_{31}{ }^{5}+{ }_{5} P_{41}{ }^{5}\right) ; \\
& W_{4}=5\left(P_{24}+P_{34}+P_{44}\right) /\left({ }_{5} P_{22}{ }^{5}+{ }_{5} P_{32}{ }^{5}+{ }_{5} P_{42}{ }^{5}\right) ; \\
& W_{5}=5\left(P_{25}+P_{35}+P_{45}\right) /\left({ }_{5} P_{23}{ }^{5}+{ }_{5} P_{33}{ }^{5}+{ }_{5} P_{43}{ }^{5}\right) \\
& W_{6}=5\left(P_{26}+P_{36}+P_{46}\right) /\left({ }_{5} P_{24}{ }^{5}+{ }_{5} P_{34}{ }^{5}+{ }_{5} P_{44}{ }^{5}\right) ; \\
& W_{7}=5\left(P_{27}+P_{37}+P_{47}\right) /\left({ }_{5} P_{25}{ }^{5}+{ }_{5} P_{35}{ }^{5}+{ }_{5} P_{45}{ }^{5}\right) ; \\
& W_{8}=5\left(P_{28}+P_{38}+P_{48}\right) /\left({ }_{5} P_{26}{ }^{5}+{ }_{5} P_{36}{ }^{5}+{ }_{5} P_{46}{ }^{5}\right) ; \\
& W_{9}=5\left(P_{29}+P_{39}+P_{49}\right) /\left({ }_{5} P_{27}{ }^{5}+{ }_{5} P_{37}{ }^{5}+{ }_{5} P_{47}{ }^{5}\right) ;
\end{aligned}
$$

where $P_{x}$ is the population in single-year completed ages $x$ and ${ }_{5} P_{x}$ is the population age range from $x$ to $x+4$. A positive (or negative) deviation above (or below) 1 for $W_{i}$ reflects digit preference (or aversion) for that respective terminal digit. A value of $W_{i}=1$ implies no age heaping. The modifications of $W_{i}$ are noted by Spoorenberg not suitable for assessing external consistency or temporal comparison. And therefore, he proposed total modified Whipple's index $\left(W_{\text {tot }}\right)$ as an overall summary index of age reporting as follows:

$$
W_{\text {tot }}=\sum_{x=0}^{9}\left|W_{i}-1\right|
$$

The summary index indicates no heaping if a value of 0 is obtained and a maximum value of 16 suggests massive heaping or poor quality of age reporting.

\section{Quality of fertility data}

Recent births and children ever born data for women aged 15-49 provide the basis for establishing a number of standard fertility measures and evaluating fertility data in censuses or surveys. Experience from African data on recent births suggests that though they are frequently underreported the emerging age pattern of fertility is usually fairly accurate (United Nations 2004). 
Symptoms of omission on parity data according to the United Nations (1983) are average parities that fail to increase rapidly enough as age increases. In some specific cases, average parities for women aged 40-44 and 45-49 may actually fall below those for women aged 35-39 even when there is no reason to suppose that fertility has been increasing. Literature suggests that the shortcomings in the completeness of reporting of parities may be achieved by using the $P / F$ ratio method of the relational Gompertz model for adjusting upwards the underreporting for births, after correcting for defective parities using the el-Badry technique (United Nations 1983, 2004; Moultrie et al. 2013).

Generally, with children ever born data, parity of childless women is often incorrectly recorded as if they had an "unknown" or a "not stated" parity. This scenario may increase average parities, especially for younger women and reduce the proportion of childless women. This becomes an issue of concern when proportion of parity in a "not stated" category is over $2 \%$ for all women in the reproductive lifespan. In such cases, the el-Badry correction is employed to detect and correct distorted parities (Moultrie et al. 2013; Schoumaker 2014; United Nations 1983, 2004).

As prior mentioned, the relational Gompertz model is regarded as a robust and the most improved $P / F$ method for preparing fertility estimates (Moultrie et al. 2013). The method provides an effective way of evaluating the extent of age and birth misreporting errors in census data as well as correcting or adjusting the fertility schedule accounting for the errors occurred. The spreadsheets "FE_Relational Gompertz" and "FE_elBadry_0" (Moultrie et al. 2013) were used to adjust agespecific fertility rates and parities.

A typical pattern for age-specific fertility rates on recent births from census data is characterised by a right-skewed concave shape showing lowest fertility rates for older ages nearing end of childbearing, lower fertility at the beginning of the reproductive lifespan for the youngest age group 15-19. In between, a peak of childbearing is observed especially prior to the age of 30 (Moultrie et al. 2013). Basing on a typical fertility curve, a number of demographic fertility models such as the Coale-Trussell and Gompertz models have been derived to fit fertility data (United Nations 1983).

Using the spreadsheet by Moultrie et al. (2013) for the relational Gompertz model, the inputs of reported age-specific fertility rates and suitably corrected average parities, using the el-Badry method, are used to generate a corrected fertility schedule which conforms to expected alpha $(\alpha)$ and beta $(\beta)$ parameter ranges. The relational Gompertz model applies in the range $-0.3<\alpha<0.3$ and $0.8<\beta<1.25$. The $\alpha$ and $\beta$ measure age location and spread of fertility schedule, respectively.

The fittings of $\alpha$ and $\beta$ were done in such as way that accurate reporting is observed when a corresponding set of $F$ value and $P$ value points strikingly linearly coincides on the same line. This also implies constant fertility when $P / F$ ratio equals 1 (or is very close to unity in real data). Possible irregularities in data occur if the $P / F$ value diverges from constant fertility. Alternatively, this implies a fertility trend-decline or increase in fertility (Brass 1996; Moultrie et al. 2013).

The consistency checks on fertility data with regard to age-sex distribution, average parities and age-specific fertility rate plausibility or expected fertility patterns are employed to assess the quality of fertility data in census data. 


\section{Results}

As a starting point, the base population data for females aged $0-80$ in Fig. 1 provide a visual display of assessing age heaping and highlighting error patterns in census data. Overall, the results show an irregular pattern of the age distribution in all the censuses reflecting a non-smooth age reporting or age heaping at ages 0 and 5 and for other terminal digits. The spikes shown in each respective census for childbearing women currently aged 15-49 are indicative of age heaping. Another explanation shows the same pattern of age heaping for the women aged 15-49 when following the same cohort from 1976 to 1986, 1997 and 2007 suggesting that the same individuals or cohorts enumerated in the subsequent census and had the same propensity to misreport in time. According to Moultrie et al. (2013), a typical population size distribution pattern for developing countries is that which shows a gradual decline with increasing age to which the results conform. Also in the entire censuses, with the exception of the 1976 census, the number of children aged 0 has been lower than those slightly older showing a decline in fertility which is underway for Swaziland.

Further applications are set out to assess the extent of age misreporting in the four censuses. The summary values of Whipple's index $(W)$ and total modified Whipple's index $\left(W_{\text {tot }}\right)$ for women in the reproductive lifespan are shown in Table 1 . The quality of age reporting for women in childbearing or reproductive lifespan has improved over time as reflected by a decrease in both Whipple's indices over time. Whipple's index (W) census value for 1976 (129.3) suggest age data reported were of rough quality (i.e. in the range 125-174.9). The values in 1986 (124.7), 1997 (118.4) and 2007 (98) indicate age reporting improved to being approximately accurate (110-124.9) and highly accurate (less than 105), respectively. A similar pattern is observed on total modified Whipple's index ( $\left.W_{\text {tot }}\right)$, a summary index which suggests the lower the values are closer to zero the less the extent of digit preference in age reporting. Thus, a value 0.7 indicates marginal or no age digit preference for the recent 2007 census. A somewhat heaping on ages appear to be increasing marginally with each preceding $(1997,1986$ and 1976) censuses. Therefore, reasonable

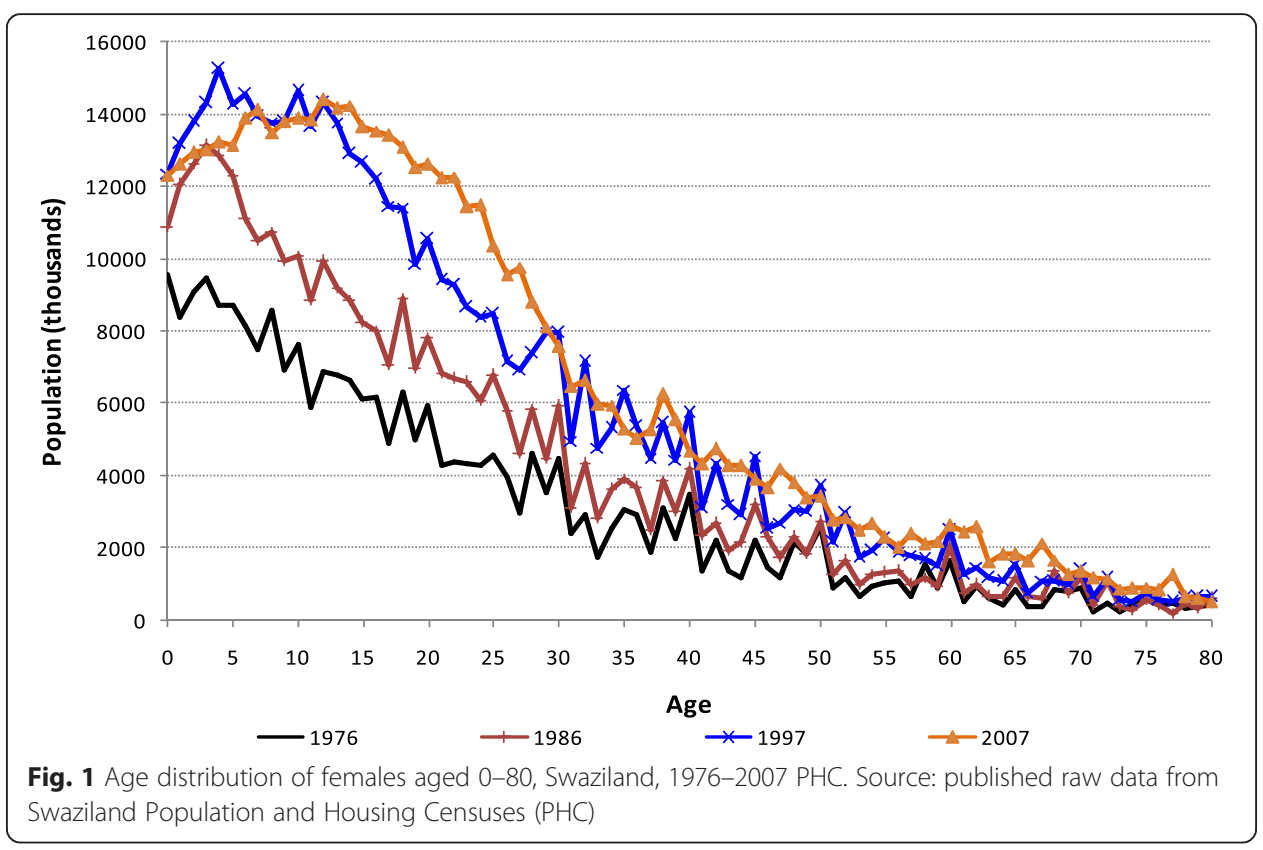


Table 1 Whipple's index $\left(W\right.$ for women aged 23-52, and total modified Whipple's index $\left(W_{\text {tot }}\right)$ for women aged 21-52, Swaziland, 1976-2007 PHC

\begin{tabular}{llr}
\hline Census & W & Wtot \\
\hline 1976 & 129.3 & 1.62 \\
1986 & 124.7 & 1.31 \\
1997 & 118.4 & 1.19 \\
2007 & 98.0 & 0.37 \\
\hline
\end{tabular}

Source: published raw data from Swaziland PHC

accuracy on all census data on age for childbearing women in Swaziland seems to be wellestablished.

For each terminal digit 0 to 9 , the pattern of digit preference (or aversion) using digit-specific modified Whipple's indices $\left(W_{i}\right)$ is shown in Fig. 2 for women in the reproductive lifespan. With the exception of the 2007 census, the findings show the significant pattern of age preference of 0, 5 and 8 in 1976, 1986 and 1997, respectively. Similarly, the pattern of age misreporting is observed with the ages ending in either digit 1 or 7 being least reported.

In addition to summary measures observed above are ratios vital in evaluation of census data. The irregular patterns of sex ratios (Fig. 3) and age ratios (Fig. 4) are indicative of errors of age misreporting, undercounting or overcounting in census data.

With regard to sex ratio, for most populations, it is expected that male mortality is little in excess of that for females as shown in Fig. 3 for Swaziland. The sex ratios at births are slightly below 100 in all the censuses indicating the possibility of underreporting of births or ages. The estimated sex ratios at birth were at 95.7, 93.3, 97.5 and 98.0 for the 1976, 1986, 1997 and 2007 censuses, respectively. The sex ratios in the middle ages, especially for ages 20-35, are overly below 100 reflecting either or a combination of excess male mortality due to HIV/AIDS, high emigration of males for labour or lower sex ratio at birth.

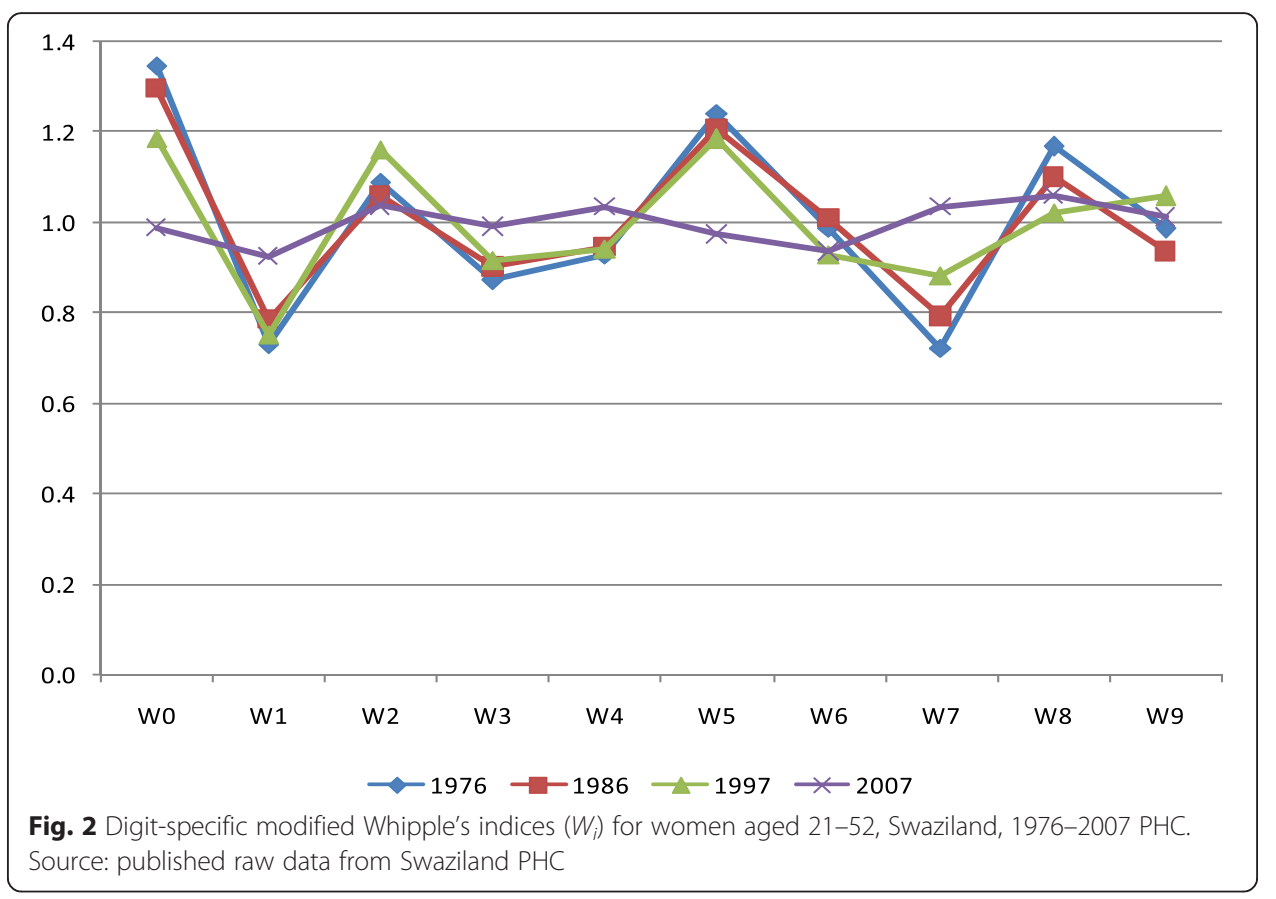




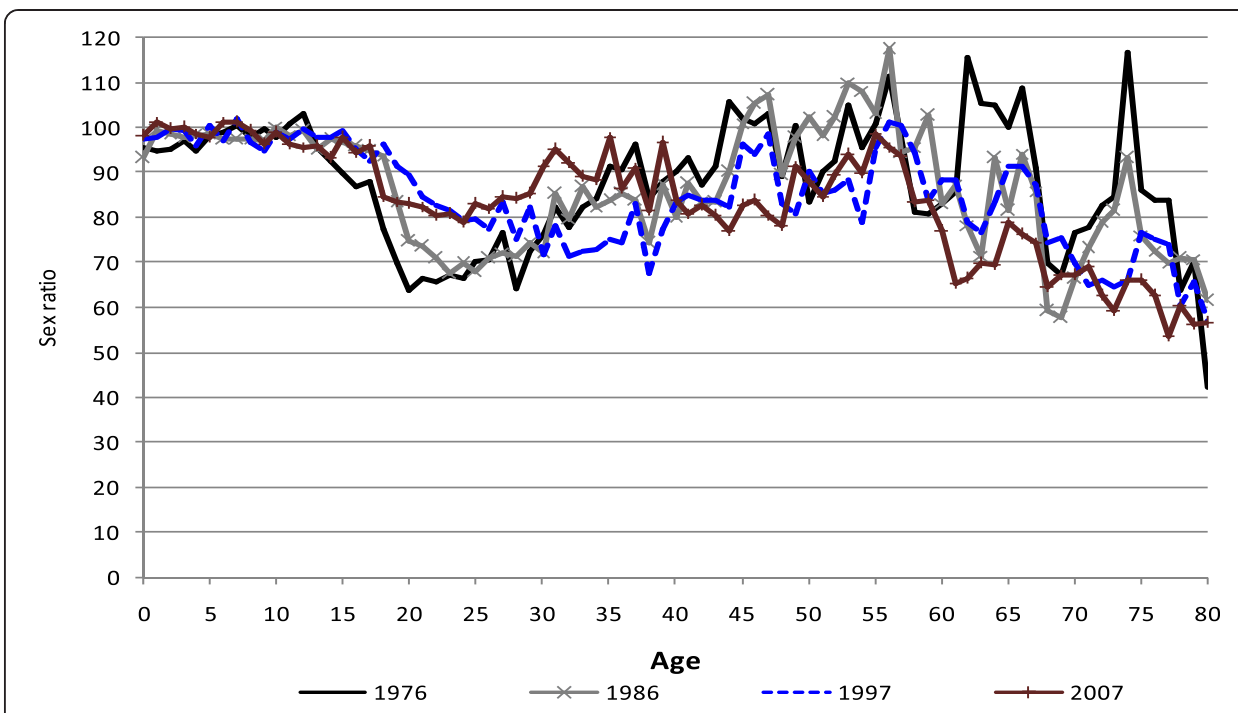

Fig. 3 Sex ratios of the population aged 0-80, Swaziland, 1976-2007 PHC. Source: published raw data from Swaziland PHC

Figure 4 tends to exhibit an irregular pattern of age ratios in all the censuses for women aged 30-49. This suggests misclassification of ages or probable undercounting for women in the middle to later childbearing years.

Employed also are rates in fertility for women aged 15-49 to detect possible anomalies in current reported fertility data. Figure 5, panel (a) to (d) shows the reported and relational Gompertz model adjusted age-specific fertility rates. The findings show underreporting of fertility or age reporting errors in all censuses for reported fertility data. However, the pattern or distribution of fertility is similar for adjusted and reported data in all four censuses. As indicated in Fig. 1 that births were declining, a comparison of the fertility rates between 1976 and 2007 in Fig. 5 confirms a decline of fertility occurring in Swaziland in the past three decades.

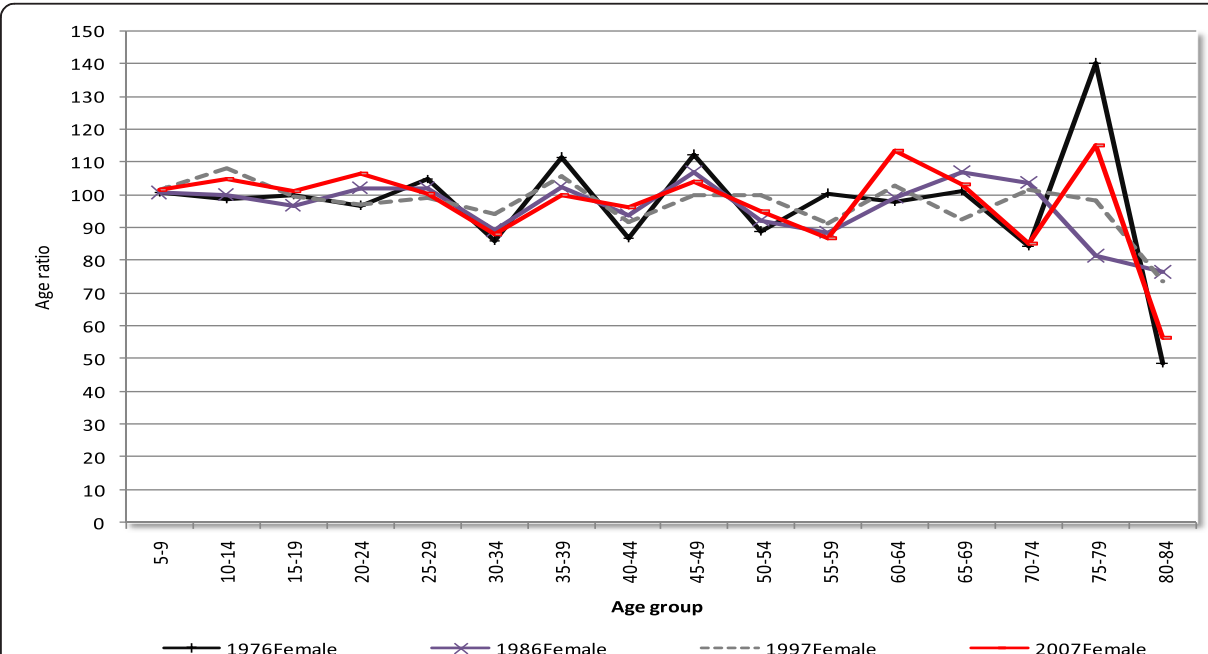

Fig. 4 Age ratio of females for 5-year age groups, Swaziland, 1976-2007 PHC. Source: published raw data from Swaziland PHC 


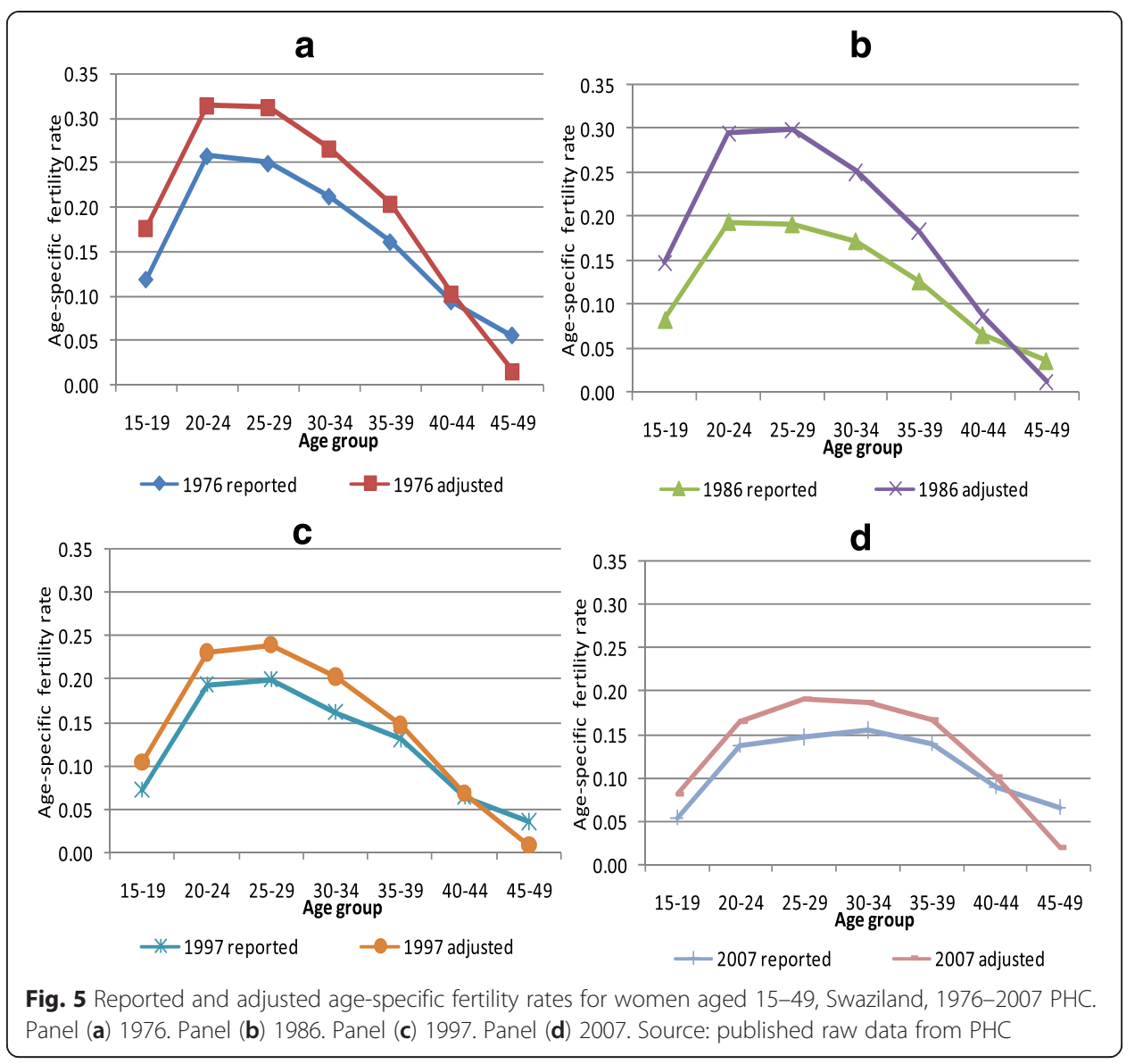

The results indicate a similar trend of peaked childbearing for women aged 20-29 in the 1976, 1986 and 1997 censuses, which is characteristic of most African countries. The 2007 census, however, illustrates a somewhat different shape of flattened peaked fertility distribution for ages 20-39. This pattern shows a similarity of fertility behaviour amongst the represented women, of which, the explanation needs further investigation. Possibly, underreporting of births may have occurred for young women aged 20-29, who normally expected to be at the peak of their childbearing. Otherwise, older women appear to have increased or just delayed childbearing.

Lifetime fertility data on parities if plotted against age group for women in childbearing would show an expected overall increasing trend as presented in Fig. 6. The reported parities for women were corrected for distorted number of parities if the overall percent of unknown parities was greater than 2, as was obtained for the 1986 and 1997 censuses. Figure 6 results reflect that the reported parities were slightly adjusted for the 1986, 1997 and 2007 censuses. The asymptotic "S" shape indicated for 1976 suggests underreporting of parities by older women or possibly rising fertility. A decrease in average parities over time suggests a fertility decline almost at all ages.

\section{Discussion and conclusion}

The study aimed at appraisal of age-sex and fertility data as components of census data used for deriving reasonable fertility estimates. Multiple census data are employed to ascertain the level of consistency in estimates or patterns. In developing countries, age 


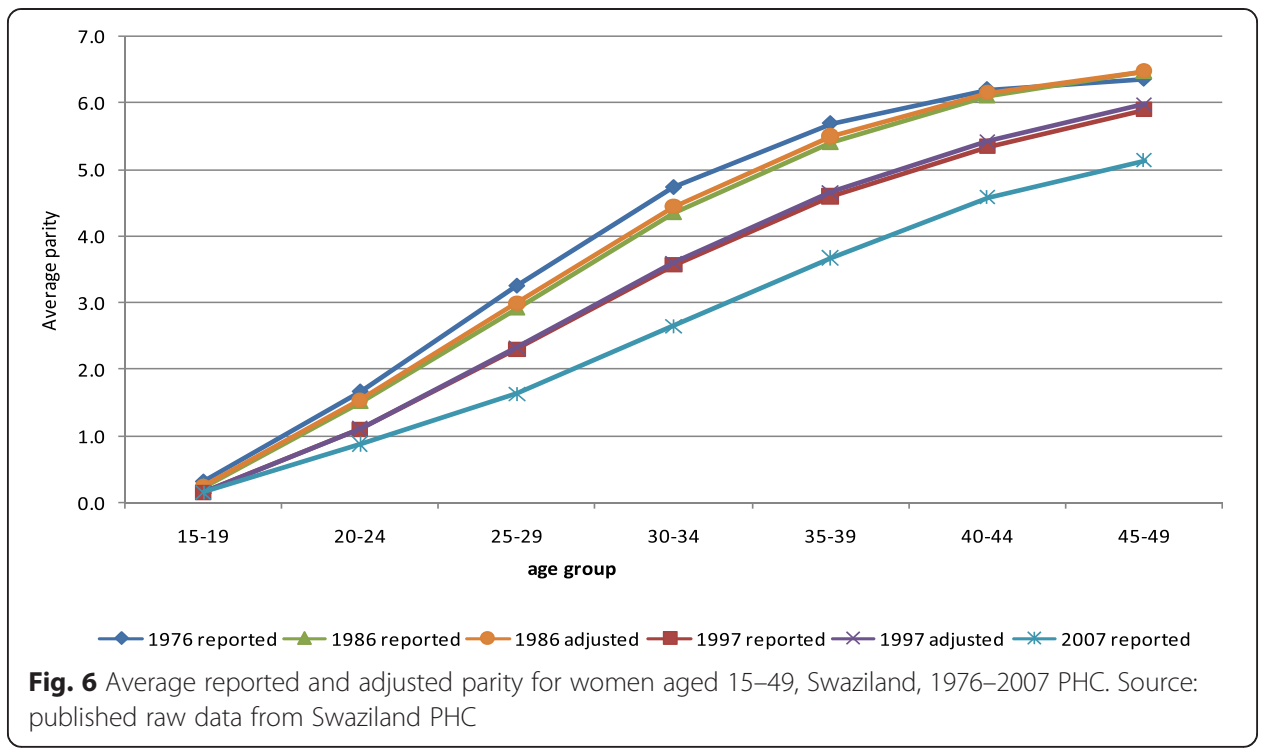

and sex data are much relied on in fertility measurement using indirect techniques of estimation. It is vital that the age-sex structure be reasonably complete and accurate to ensure quality on the resulting estimates of basic demographic indices.

According to Moultrie et al. (2013), the basis of such enquiry looks at dimensions on data of age-sex structure, current fertility and lifetime fertility (or average parity) patterns as employed in this paper. Accordingly, consistency checks on the expected or plausible patterns with regard to the three stated dimensions were done using published data from the 1976, 1986, 1997 and 2007 Swaziland censuses. As a caution or a limitation, reported or published data utilised could have been edited or manipulated, but the extent of such manipulation cannot be verified when raw data are unavailable (e.g. 1976 and 1986 censuses).

The population distribution, Myers' index, Whipple's index, age ratios and sex ratios were instrumental in checking for consistency or anomalies in the multiple census data. The assessment of the age and sex distribution for all the four censuses using the above-mentioned demographic tools yielded a number of observations.

The quality of age reporting in the 1976 and 1986 censuses is moderate or reasonable. In three decades, from 1976 to 2007, the quality of age data in Swaziland improved from rough to highly accurate using Whipple's indices criteria. Both Whipple's index and total modified Whipple's index showed consistency in detecting age preference. The preference for ages ending with digit 0 and 5 including 8 for Swaziland decreased drastically over time. This marked decrease was seen also for digits 1,3 , and 7, which had the highest avoidance. No significant differentials in the age reporting between censuses were evident in the census data.

Overall, a consistent irregular pattern of age ratios and sex ratios in singles years of ages in all four censuses reflects age misreporting often due to understating or overstating of ages. Its effect on fertility is uncertain (United Nations 1983).

The jagged pattern of age ratios suggests age exaggeration or displacement. Affirming this is the possible underreporting of children as reflected in the age structure, which begins with an "L", shaped pattern or depression for single-year ages. The age distribution 
in single years of population also confirms age misreporting through age heaping or digit preference. The lesser extent of digit preference for the recent 2007 and 1997 censuses compared to the earlier 1986 and 1976 censuses reflects an improvement in the quality of age reporting in the three decades. This may be linked to possibly many factors such as improvement in the education of the populace or data collection procedures in the context of Swaziland.

Swaziland appears to have lower sex ratios at birth (above 90 but less than 100) in all censuses suggesting an undercount of male births if not excess male mortality. This is uncommon in most world population but typical for some African countries (Garenne 2004; Moultrie et al. 2013).

The lower sex ratios at birth for Swaziland, much lower than 103, the average for Africans (Garenne 2004), are characteristic of Eastern and Southern African countries. Thus, according to Garenne (2004), a low value within the range of $0.87-1.03$, as found in the censuses, shows good data.

Recent births and parity data collected from censuses often are limited or inaccurate requiring the use of corrective demographic techniques (United Nations 1983). Reported data on the average parities appears to be increasing with women's age as expected in all censuses, although underreporting of births for older women is much poorer in 1976 in particular probably due to lower levels of education attained by women then. Similarly, the age pattern of fertility appears to be plausible showing a concave shape as expected with the exception of the 2007 census where a flat topped fertility pattern is observed.

However, applying the relational Gompertz technique, a modified $P / F$ ratio method using the reported seemingly less fault current fertility and parity data shows fertility is underestimated in Swaziland. Thus, adjustment demographic methods when applied correctly are imperative in evaluating and adjusting demographic data as supported by Hill (1990). For older women aged 45-49 in all the censuses, the reported age-specific fertility rates were seemingly higher than those adjusted, which reflects faulty age reporting for older women.

Estimates of fertility may be flawed by certain degree of errors in reporting of ages and births. The analysis of results indicates some irregularities in age structure in all the four censuses of Swaziland. The irregularities in the age distribution reflected in the sex ratios may reflect net migration for young adults.

The extent of age reporting errors in census data is moderate as evaluated as shown in the several methods applied for the four census data and therefore estimates of fertility from the census data can be derived of reasonable quality. Hence, methods of fertility measurement using the reported parity-fertility data and age-sex population structure can be dependable when best methods and robust assumption on generating fertility estimates are made. However, their usefulness becomes questionable when agesex structure and fertility data utilised are defective and incomplete, of which the study found otherwise.

Actual raw data were not available for the study, except for the 1997 census which was correctly verified with the published census data. The limitation on the published data is that further manipulation and analysis of data was limited. The available raw data requires that data documentation and archiving practice standards should be implemented addressing the processing of data such as on editing. 
Competing interests

The authors declare that they have no competing interests.

\section{Authors' contributions}

GBC and CO conceived the study. GBC and ENZ collected the data. GBC drafted the Introduction, Data and methods, and Discussion and conclusion. JDG drafted the Results and Discussion. CO and ENZ revised the manuscript for important intellectual content. All authors analysed data, interpreted the results, read and approved the final manuscript.

\section{Authors' information}

GBC is a lecturer in the Department of Statistics and Demography at the University of Swaziland (UNISWA). He is currently studying as a doctoral candidate in the Demography and Population Studies (DPS) programme at the University of the Witwatersrand. JDG is a doctoral candidate of DPS. ENZ is a senior lecturer in the Department of Statistics and Demography at UNISWA. Co is a Professor and head of DPS programme at the University of the Witwatersrand.

\section{Acknowledgements}

The authors acknowledge the Central Statistics Office (CSO) of Swaziland support for providing the census data and reports used in this study. The authors are grateful to the Department of Higher Education and Training grant of the Faculty of Humanities, University of the Witwatersrand, for the financial support to organise the writing retreat at which this manuscript was prepared. The support of the DST-NRF Centre of Excellence in Human Development towards the research is hereby acknowledged. Opinions expressed and conclusions arrived at are those of the authors and not necessarily to be attributed to either the Centre of Excellence in Human Development or CSO.

\section{Received: 11 September 2015 Accepted: 7 January 2016}

\section{Published online: 14 July 2016}

\section{References}

Blanc, A. K., \& Rutstein, S. O. (1994). The demographic transition in Southern Africa: yet another look at the evidence from Botswana and Zimbabwe. Demography, 31(2), 209-215.

Brass, W. (1996). Demographic data analysis in less developed countries: 1946-1996. Population Studies, 50(3), $451-467$.

Cleland, J. (1996). Demographic data collection in less developed countries. Population Studies, 50(3), 433-450.

Cohen, B. (1993). Fertility levels, differentials, and trends. In K. A. Foote, K. H. Hill, \& L. G. Martin (Eds.), Demographic change in sub-Saharan Africa (pp. 8-67). Washington DC: National Academy Press.

Cohen, B. (1998). The emerging fertility transition in sub-Saharan Africa. World Development, 26(8), 1431-1461.

Garenne, M. (2004). Sex ratios at birth in populations of Eastern and Southern Africa. Southern African Journal of Demography, 9(1), 91-96.

Hill, K. (1990). Demographic estimation from deficient or defective data. In B. Fetter (Ed.), Demography from scanty evidence: central Africa in the colonial era (pp. 133-142). Boulder: L. Rienner Publishers.

Joyner, K., Peters, H. E., Hynes, K., Sikora, A., Taber, J. R., \& Rendall, M. S. (2012). The quality of male fertility data in major U.S. surveys. Demography, 49(1), 101-124.

Moultrie, T. A., \& Timæus, I. M. (2002). Trends in South African fertility between 1970 and 1998: an analysis of the 1996 Census and the 1998 Demographic and Health Survey. http://www.mrc.ac.za/bod/trends.pdf. Accessed 21 May 2015.

Moultrie, T. A., Dorrington, R. E., Hill, A., Hill, K., Timæus, I., \& Zaba, B. (2013). Tools for demographic estimation. Paris: International Union for the Scientific Study of Population.

National Research Council. (2004). The 2000 Census: Counting Under Adversity. Panel to Review the 2000 Census (Committee on National Statistics, Division of Behavioral and Social Sciences and Education). Washington: The National Academies Press.

Phillips, H. (1999). Demographic survey data in South Africa: an evaluation of methodology and quality. Southern African Journal of Demography, 7(1), 1-10.

Potter, J. E. (1977). Problems in using birth-history analysis to estimate trends in fertility. Population Studies, 31(2), 335-364.

Schoumaker, B. (2014). Quality and consistency of DHS fertility estimates, 1990 to 2012 (DHS Methodological Reports No. 12). Rockville: ICF International.

Shryock, H. S., Siegel, J. S., et al. (1976). The methods and materials of demography. (Condensed ed., studies in population). New York: Academic Press, Inc.

Spoorenberg, T. (2007). Quality of age reporting: extension and application of the modified Whipple's Index. Population (English edition), 4(62), 729-741.

Spoorenberg, T. (2014). Reverse survival method of fertility estimation: an evaluation. Demographic Research, 31(9), 217-246.

Thomas, D., \& Muvandi, I. (1994). The demographic transition in Southern Africa: reviewing the evidence from Botswana and Zimbabwe. Demography, 31(2), 217-227.

United Nations. (1983). Manual X: indirect techniques for demographic estimation. New York: United Nations.

United Nations. (2004). Handbook on the collection of fertility and mortality data. http://unstats.un.org/unsd/ demographic/standmeth/handbooks/Handbook_Fertility_Mortality.pdf. Accessed 21 May 2015.

Vergauwen, J., Wood, J., De Wachter, D., \& Neels, K. (2015). Quality of demographic data in GGS wave 1. Demographic Research, 32(24), 723-774. 